\title{
Influência de Diferentes Condições Higrotérmicas na Resistência à Tração de Compósitos de Fibra de Carbono/Epóxi Modificada
}

\author{
José Antônio P. Cunha \\ Departamento de Engenharia Aeronáutica e Mecânica, ITA/SENAI \\ Michelle L. Costa, Mirabel C. Rezende \\ Divisão de Materiais, IAE, CTA
}

\begin{abstract}
Resumo: Este trabalho foi direcionado para a análise dos efeitos higrotérmicos na resistência à tração de compósitos poliméricos termorrígidos. Foram investigados os efeitos da umidade e da temperatura sobre as resistências à tração longitudinal e à tração transversal de compósitos ([0/0]s) de fita de carbono unidirecional impregnada com resina epóxi 8552. Os ensaios de resistência à tração longitudinal, realizados à temperatura ambiente, mostraram que as amostras não perderam sua resistência quando condicionadas. Porém, os compósitos apresentaram uma redução na sua resistência quando submetidos à câmara de climatização $\left(80^{\circ} \mathrm{Ce} 90 \%\right.$ de umidade) e ensaiados à temperatura elevada. Já nos ensaios de tração transversal foi observado que as amostras submetidas à câmara de névoa salina e ensaiadas à temperatura ambiente apresentaram uma pequena redução na sua resistência, porém as amostras submetidas à câmara de climatização mostraram uma redução acentuada na resistência As amostras ensaiadas à temperatura elevada e submetidas à câmara de névoa salina apresentaram uma pequena diminuição na sua resistência (11\%). Já uma acentuada diminuição da resistência à tração transversal (51\%) foi observada quando as amostras de compósitos poliméricos foram submetidas à câmara de climatização e ensaiadas à temperatura elevada. Também foram obtidas fotomicrografias via microscopia eletrônica de varredura (MEV) das seções de fraturas das amostras ensaiadas em tração em todos os tipos de condicionamento estudados no presente trabalho. Todos estes fatos mostraram que a matriz polimérica é afetada nos ensaios à temperatura elevada.
\end{abstract}

Palavras-chave: Compósito polimérico, fibra de carbono, epóxi, resistência à tração, efeito higrotérmico.

\section{Influence of Different Hygrothermal Conditions on the Tensile Strength of Carbon/epoxy 8552 Composites}

Abstract: This work focused the analysis of hygrothermal ageing effects on the tensile strength of thermoset laminated composites. Humidity and temperature effects on the longitudinal and transversal tensile strengths of carbon fiber/epoxy 8552 unidirectional laminates tape composites ([0/0]s) were investigated. The longitudinal tensile strength results at room temperature was not significantly affected by exposing the samples to either the humidity-controlled chamber or the salt spray chamber. However, the specimens submitted to the humidity-controlled chamber and tested at elevated temperature $\left(80{ }^{\circ} \mathrm{C}\right.$ and $\left.90 \% \mathrm{RH}\right)$ presented a decrease in the longitudinal tensile strength. A small decrease in the transversal tensile strength results was observed during room temperature tests in the samples submitted to salt spray chamber. A higher decrease in the transversal tensile strength was verified in the samples submitted to the humidity-chamber. Samples submitted to the salt spray chamber tested at elevated temperature showed a small decrease in the transversal tensile strength (11\%). A drastic reduction in the strength (51\%) was observed in the samples submitted to the humidity-chamber and tested at high temperature. A fractograph study by scanning electronic microscopy (SEM) was performed in every single sample involved in the tests. These results highlight that the elevated temperatures affect the polymeric matrix.

Keywords: Carbon-fiber, epoxy laminate composite, hygrothermal ageing, tensile properties.

\section{Introdução}

Atualmente, a variedade de materiais disponíveis para uso em engenharia é extraordinariamente grande, atendendo às mais diversas aplicações do mercado. Dentro desse contexto, os compósitos poliméricos apresentam-se como um caso de reconhecido interesse em materiais não convencionais de engenharia ${ }^{[1-4]}$.

Os compósitos são materiais de engenharia geralmente constituídos por reforço, com orientação definida ou não, disperso em uma matriz metálica, cerâmica ou polimérica. No caso dos compósitos poliméricos o reforço fibroso é o

Autor para correspondência: Mirabel C. Rezende, Divisão de Materiais, IAE, CTA, Praça Marechal do Ar Eduardo Gomes no 50 - Vila das Acácias, CEP: 12228-904, São José dos Campos, SP. E-mail: mirabel@iae.cta.br 
principal constituinte e ocupa a maior fração de volume do material, sendo responsável pela resistência ao carregamento mecânico e tem maior influência sobre as propriedades mecânicas. Seu desempenho estrutural depende da disposição das fibras em uma ou mais direções, empilhadas em uma seqüência de camadas definidas no projeto do compósito, a fim de se obter produtos com valores de resistência desejáveis ${ }^{[5-7]}$.

No caso de compósitos poliméricos, outro importante constituinte é a matriz polimérica. Esta tem as funções de agregar as fibras, dar proteção às suas superfícies contra danos por abrasão e atenuar os efeitos adversos das condições ambientais na utilização do compósito. A matriz deve apresentar uma adesão adequada à superfície do reforço, de tal forma que no carregamento mecânico aplicado a um componente, a matriz se deforme e transfira a carga entre as fibras contínuas e/ou descontínuas do compósito. Também, as propriedades de resistência à compressão, resistência ao cisalhamento interlaminar, resistência ao cisalhamento no plano e a temperatura de serviço do componente são dominadas pelo sistema de matriz polimérica ${ }^{[4,8,9]}$.

Existe um elevado grau de versatilidade nas propriedades mecânicas dos materiais compósitos reforçados com fibras contínuas. A resistência e a rigidez mecânica podem ser variadas significativamente em diferentes áreas do compósito pela seleção do tipo, da forma, da orientação apropriada das fibras e pelo controle do local de concentração da fibra. Além disso, as propriedades físicas dos compósitos de fibra de carbono, tais como a sua estabilidade térmica, condutividade elétrica e resistência à corrosão podem ser modificadas pela variação das condições de processamento do compósito e por uma escolha apropriada do material da matriz ${ }^{[3]}$.

Os compósitos poliméricos, quando submetidos a condições de serviço, são expostos também a uma variedade de condições ambientais e diversos tipos de carregamentos mecânicos. Os principais agentes atmosféricos causadores dos ataques ambientais são a temperatura; umidade relativa do ar; efeitos de radiação ultravioleta; ataque de partículas do espaço; exposição química; água salina; combustível; gases e fluidos hidráulicos. Já as tensões mecânicas são originadas dos esforços que atuam na estrutura como, por exemplo: tração, compressão e cisalhamento. Estas exposições do meio ambiente combinada com as deformações e tensões mecânicas que atuam nas estruturas são as condições em que os compósitos poliméricos termorrígidos devem atuar ${ }^{[10]}$.

A polaridade da matriz tem grande influência na absorção de água pelo compósito. Quanto maior for a polaridade da matriz maior será a sua capacidade de absorção de água. As matrizes epóxi, por exemplo, que curam por meio de grupos hidroxilas e amínicos, possuem em sua estrutura tridimensional grupos fortemente polares $(\mathrm{OH})$ que podem facilmente se ligar a moléculas de água por meio de ligações de hidrogênio. Assim, a adição de diluentes reativos não-polares em matrizes polares leva a uma diminuição de absorção de umidade no compósito ${ }^{[11-13]}$.

Além das interações polares entre as moléculas de água e a resina, a configuração espacial da molécula de resina, utilizada como matriz polimérica, também tem influência no processo de absorção de umidade pelo material. Diferentes configurações geométricas espaciais podem resultar em uma estrutura mais aberta, o que favorece a absorção de umidade pela cadeia polimérica, pois a água pode se alojar nos interstícios da estrutura da molécula. Conseqüentemente, polímeros com um arranjo cristalino bem empacotado são mais eficientes na resistência à umidade que aqueles com menor grau de empacotamento. Assim alguns pesquisadores têm tentado correlacionar a absorção de umidade dos materiais com o volume livre existente dentro das moléculas dos mesmos, porém esse estudo ainda não está bem consolidado ${ }^{[11]}$.

Embora a natureza da matriz seja o fator dominante no processo de absorção, o tipo de fibra, a orientação das fibras, a seqüência de empilhamento das camadas e o tipo de acabamento da borda livre do laminado podem influenciar no processo de difusão da umidade no compósito ${ }^{[14-16]}$.

De acordo com as considerações normalmente encontradas na literatura, a umidade absorvida nos compósitos de fibras de carbono/epóxi pode reduzir a resistência e a rigidez do laminado, devido à plasticização da matriz com o enfraquecimento da interface fibra/resina ${ }^{[19]}$. Essas reduções são particularmente significativas em temperaturas elevadas. Os compósitos poliméricos com a superfície de fibras expostas são citados como evidências do enfraquecimento da interface fibra/resina devido ao ambiente úmido ${ }^{[20]}$. Assim, a presença da umidade no compósito pode causar mudanças marcantes nas características físicas e químicas da resina. Em uma resina epóxi, decréscimos no módulo de elasticidade e redução na temperatura de transição vítrea são evidências do mecanismo de plasticização da matriz ${ }^{[21,22]}$. Nesse caso, quando se deseja utilizar as propriedades do material por um longo período de tempo torna-se necessário considerar a ação desse mecanismo de degradação da matriz polimérica.

Foi observado na literatura que, para muitas matrizes, o efeito do aumento da umidade é muito similar ao aumento da temperatura. Collings; Harvey e Dalziel ${ }^{[23]}$ demonstraram experimentalmente que um incremento de temperatura de $70{ }^{\circ} \mathrm{C}$ produz um efeito equivalente ao da absorção de umidade na resistência à compressão, tração e ao cisalhamento interlaminar, reproduzindo o modo de falha característico de estruturas submetidas ao calor/umidade. A vantagem desse método foi a de eliminar a necessidade de condicionar o compósito em ambientes úmidos para se conhecer a influência dos parâmetros calor/umidade no material em estudo.

Os efeitos ambientais causados pela temperatura e umidade relativa do ar podem ser reversíveis quando o período de exposição é de curta duração. Porém, quando a exposição ocorre em ciclos prolongados, onde a combinação da umidade com mudança de temperatura está presente, os efeitos produzidos podem ser irreversíveis devido à afinidade da água por grupos funcionais específicos de matrizes poliméricas de natureza polar. Nesse caso, geralmente ocorrem alterações destrutivas na interface reforço/matriz polimérica, devido à degradação das prováveis interações físico-químicas existentes entre a resina e a fibra. Em conseqüência, ocorre o 
descolamento da fibra, provocando a delaminação do compósito com a conseqüente redução das propriedades mecânicas e termofísicas do material compósito ${ }^{[10-18]}$.

O presente estudo foi direcionado para a análise dos efeitos higrotérmicos sobre a resistência à tração de compósitos poliméricos termorrígidos. Foram investigados os efeitos da umidade e de temperatura sobre as resistências à tração longitudinal e à tração transversal de compósitos de fita carbono unidirecional impregnados com resina epóxi (F8552), submetidos aos efeitos de condicionamento ambiental.

\section{Experimental}

\section{Materiais}

Os compósitos utilizados neste estudo foram obtidos a partir de reforços pré-impregnados na forma de fitas unidirecionais de carbono/epóxi (F8552), de onde foram preparados os corpos-de-prova (cdp's) para os ensaios de resistências à tração longitudinal e à tração transversal, às temperaturas elevada $\left(82 \pm 1{ }^{\circ} \mathrm{C}\right)$ e ambiente $\left(22 \pm 3{ }^{\circ} \mathrm{C}\right)$. As fitas unidirecionais foram empilhadas umas sobre outras mantendo mesma orientação, ou seja, zero em relação às fibras $[(0)]_{\mathrm{S}} . \mathrm{O}$ sistema de resina epóxi 8552 é formado pela tetra glicidil metileno bis-anilina do bisfenol-A curado com diamina aromática ${ }^{[24]}$

O reforço dos pré-impregnados (prepregs) utilizados para a fabricação dos cdp's avaliados neste trabalho foi a fibra de carbono de alta resistência à tração $(5,76 \mathrm{MPa})$, possuindo massa específica de $1,79 \mathrm{~g} / \mathrm{cm}^{3}$, deformação máxima de $1,99 \%$, módulo de $290 \mathrm{GPa}$ e previamente tratada para uma maior compatibilidade química com a resina epóxi modificada, de alta resiliência e resistência ao impacto. Este tipo de fibra tem a denominação de IM7. Todos os pré-impregnados utilizados neste trabalho foram produzidos pela empresa Hexcel Composites e fornecidos pela EMBRAER na forma de carretel de fita unidirecional contínua (tape) de carbono com resina epóxi 8552.

Todos os laminados foram curados em autoclave de acordo com o ciclo de cura sugerido pelo fornecedor dos préimpregnados, sendo o mesmo utilizado para a consolidação dos laminados de fibra de carbono/epóxi 8552 dentro da

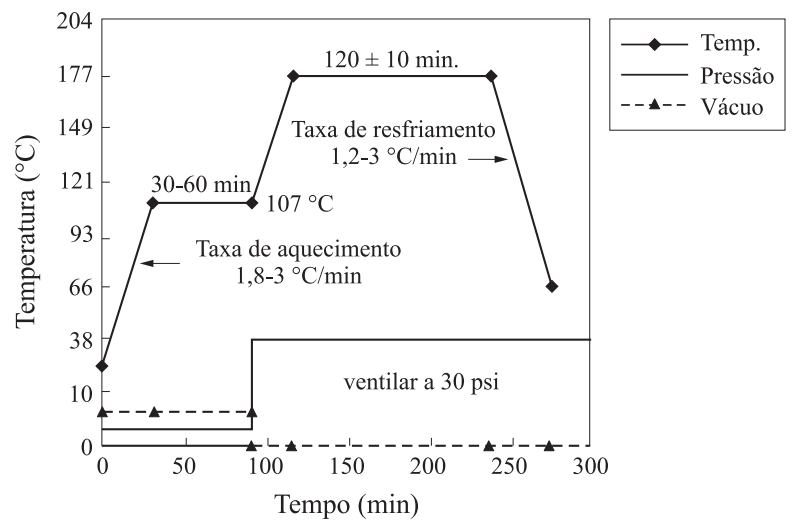

Figura 1. Ciclo de cura em autoclave utilizado na cura da resina epóxi F8552
EMBRAER. A autoclave é um vaso de pressão que permite a aplicação de forma combinada de pressão, calor e vácuo para a consolidação das reações de reticulação da resina transformando-a em um termorrígido.

O ciclo de cura (Figura 1) é composto de 5 etapas: aquecimento até $116^{\circ} \mathrm{C}$ à razão de aquecimento de $2,5^{\circ} \mathrm{C} / \mathrm{min}$; isoterma a $116^{\circ} \mathrm{C}$ por $60 \mathrm{~min}$; aquecimento de $116^{\circ} \mathrm{C}$ até $177^{\circ} \mathrm{C}$ à razão de aquecimento de $2,5^{\circ} \mathrm{C} / \mathrm{min}$, isoterma a $177^{\circ} \mathrm{C}$ por $120 \mathrm{~min}$ e resfriamento até $65^{\circ} \mathrm{C}$ à razão de resfriamento de $2.5^{\circ} \mathrm{C} / \mathrm{min}$. A aplicação da pressão de $0,69 \mathrm{MPa}$ foi realizada a $116^{\circ} \mathrm{C}$.

Os cdp's obtidos encontram-se descritos nas Tabelas 1 e 2 .

\section{Condicionamento ambiental}

Para avaliar o efeito de condicionamento ambiental em compósitos poliméricos três amostras de acompanhamento, doze de tração transversal e doze de tração longitudinal foram submetidas às condições higrotérmicas. Os condicionamentos dos cdp's foram obtidos através da exposição higrotérmica em água destilada em uma câmara de climatização. Outro conjunto de amostras foi condicionado em água salina através da exposição do material a uma solução de cloreto de sódio $(\mathrm{NaCl})$ em uma câmara de névoa salina, também conhecida como salt spray.

O condicionamento em câmara de climatização é um teste gravimétrico que controla a alteração do ganho de umidade no compósito em função do tempo, pela medida da mudança de massa em amostras de acompanhamento, até atingir o estado efetivo de equilíbrio de umidade na estrutura do compósito.

Antes de iniciar a climatização, todos os cdp's e amostras de acompanhamento foram secos em estufa a $60 \pm 2{ }^{\circ} \mathrm{C}$ por quatro horas. Após esse período as amostras de acompanha-

Tabela 1. Matriz de teste dos cdp's de resistência à tração transversal.

\begin{tabular}{cccc}
\hline Condicionamento & $\begin{array}{c}\text { Laminado } \\
\left(\mathbf{9 0}^{\mathbf{0}}\right)\end{array}$ & cdp's & $\begin{array}{c}\text { Propriedade ensaiada } \\
\text { à temp. ambiente e à } \\
\text { temp. elevada }\end{array}$ \\
\hline Seco & Unidirecional & 6 & Tração transversal \\
$\begin{array}{c}\text { Câmara de } \\
\text { climatização } \\
\text { Câmara de } \\
\text { névoa salina }\end{array}$ & Unidirecional & 6 & Tração transversal \\
\hline temp. - temperatura & & 6 & Tração transversal \\
\hline
\end{tabular}

Tabela 2. Matriz de teste dos cdp's de resistência à tração longitudinal.

\begin{tabular}{cccc}
\hline Condicionamento & $\begin{array}{c}\text { Laminado } \\
\left(\mathbf{0}^{\mathbf{*}}\right)\end{array}$ & cdp's & $\begin{array}{c}\text { Propriedade ensaiada } \\
\text { à temp. ambiente e à } \\
\text { temp. elevada }\end{array}$ \\
\hline Seco & Unidirecional & 6 & Tração longitudinal \\
$\begin{array}{c}\text { Câmara de } \\
\text { climatização } \\
\begin{array}{c}\text { Câmara de } \\
\text { névoa salina }\end{array}\end{array}$ & Unidirecional & 6 & Tração longitudinal \\
\hline temp. - temperatura & & 6 & Tração longitudinal \\
\hline
\end{tabular}


mento foram rapidamente removidas da estufa para um dessecador até que atingissem a temperatura ambiente e fossem pesadas em balança analítica, marca Mettler-Toledo modelo AB-204-S, com precisão de $\pm 0,1 \mathrm{mg}$, enquanto os cdp's permaneciam na estufa. Este procedimento foi realizado com base na norma ASTM C $562-85^{[14]}$ e a operação repetida várias vezes até que a massa seca das amostras ficasse constante, com precisão de $\pm 0,002 \mathrm{~g}$. Em seguida, esses cdp's e amostras de acompanhamento foram transferidos para as suas respectivas câmaras de condicionamento e iniciados os ensaios.

\section{Câmara de climatização}

A exposição higrotérmica em câmara de climatização foi baseada no Procedimento B da norma ASTM D 5229/D5229 $M-92^{[15]}$ para materiais compósitos a serem submetidos a ensaios mecânicos na condição úmida. As amostras de acompanhamento (sem tabs) e os cdp's foram expostos à temperatura de $80^{\circ} \mathrm{C}$, que é o máximo valor recomendado pela norma para matriz epóxi curada a $177^{\circ} \mathrm{C}$. O teor de umidade relativa escolhido foi de $90 \%$ por um período de 9 semanas (período para se atingir a saturação de umidade). Estes parâmetros foram programados em uma câmara de condicionamento higrotérmico Climats-Sapratin - Modelo Excal 2214-HE, calibrada para manter por monitoração automática o valor da temperatura na faixa de $\pm 1{ }^{\circ} \mathrm{C}$ e o teor de umidade relativa na faixa de $\pm 1 \%$.

\section{Câmara de névoa salina (salt spray)}

O condicionamento em câmara de névoa salina foi realizado segundo a norma ASTM $117-03^{[16]}$ para verificar a resistência dos cdp's em condição salina por um período de sete semanas. As amostras de acompanhamento e os cdp's foram suspensos entre $15^{\circ}$ e $30^{\circ}$ na vertical paralela à direção principal do fluxo horizontal de névoa dentro da câmara, posicionados de forma a não permitir que houvesse contato entre eles. A solução salina foi preparada pela dissolução de 5 partes de cloreto de sódio em 95 partes $(\mathrm{m} / \mathrm{m})$ de água destilada com $\mathrm{pH}$ na faixa de 6,5 a 7,2. Os cdp's foram expostos à temperatura de $35^{\circ} \mathrm{C}$, sendo que para obter a saturação da solução salina a temperatura é de $46^{\circ} \mathrm{C}$ e pressão de $9,81 \mathrm{~N} / \mathrm{cm}^{2}$ (valores recomendados pela norma).

\section{Resistências à tração longitudinal e transversal}

As geometrias dos cdp's utilizados nos ensaios de resistência à tração foram adotadas conforme a norma ASTM D 3039/D 3039M-00 [17], que determina que os cdp's tenham uma espessura entre $(0,508$ a 2,54$) \mathrm{mm}$, uma largura de $12,7 \mathrm{~mm}$ e um vão de no mínimo $127 \mathrm{~mm}$ para os ensaios de tração longitudinal, onde a aplicação da carga é na orientação normal do compósito (através da espessura) [(0)] . Para os ensaios de tração transversal, uma largura de $25,4 \mathrm{~mm}$ e comprimento útil mínimo de $38,1 \mathrm{~mm}$, com a carga aplicada ortogonalmente à orientação do plano dos compósitos $[(90)]_{\mathrm{s}}$. Os tabs para fixação na máquina de ensaio são de fibra de vidro medindo $(60 \times 12,75 \times 7,4) \mathrm{mm}$ para a tração longitudinal

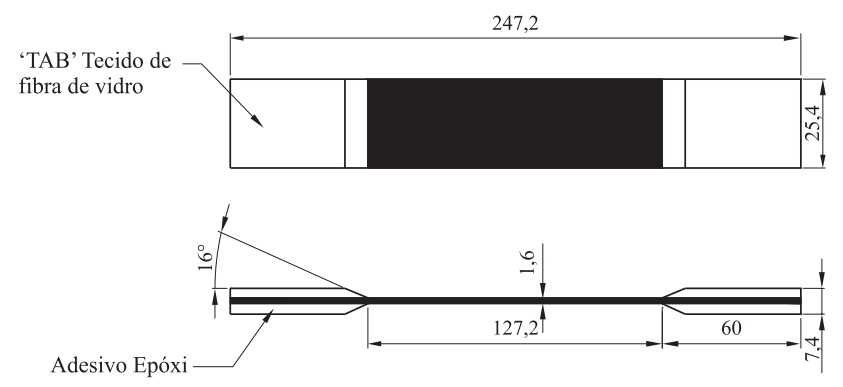

Figura 2. Esquema representativo das dimensões dos cdp's utilizados nos ensaios de tração transversal.

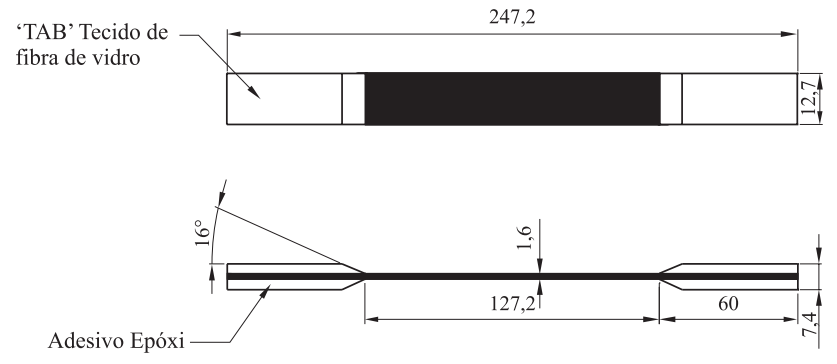

Figura 3. Esquema representativo das dimensões dos cdp's utilizados nos ensaios de tração longitudinal.

e $(60$ x 25,4 x 7,4)mm para a tração transversal sendo colados nas extremidades dos cdp's. Os tabs são colados com filme adesivo para reduzir a concentração de tensões na interface de colagem, distribuir homogeneamente as forças de agarramento na fixação do corpo-de-prova na máquina de ensaio e proteger a superfície do laminado contra danos. Os tabs são obtidos a partir de laminados de vidro/epóxi, que devido ao baixo módulo de elasticidade do compósito, deformam-se elasticamente distribuindo tensões por uma grande área e absorvendo energia. Para cada família de compósito foram avaliadas 6 amostras com dimensões de $(247,2$ x 25,4 $\mathrm{x} 1,6) \mathrm{mm}$, sendo comprimento $\mathrm{x}$ largura $\mathrm{x}$ espessura, para a tração transversal (Figura 2) e (247,3 x 12,7 x 1,6)mm para a tração longitudinal (Figura 3).

\section{Resultados e Discussão}

\section{Absorção de umidade}

O ganho na porcentagem do teor de umidade pode ser medido gravimetricamente, isto é, com a mudança na massa da amostra polimérica. O conteúdo de umidade absorvido pelas famílias de compósitos unidirecionais de fibras de carbono IM7 impregnadas com resina epóxi 8552 está apresentado na Tabela 3, como resultado do ganho de massa médio obtido semanalmente nas amostras de acompanhamento.

Observa-se, nas Figuras 4 e 5, que no estágio inicial, existe uma relação praticamente linear entre a absorção de umidade e a raiz quadrada do tempo. Por curtos períodos de tempo, o conteúdo de umidade aumenta linearmente até atingir um estado conhecido como pseudo-equilíbrio, aproximadamente entre três e quatro semanas de exposição. Esse estágio de pseudo-equilíbrio é praticamente o mesmo para a maioria dos compósitos poliméricos termorrígidos, pois isso é característico de comportamento de absorção de umidade segundo a lei 
Tabela 3. Resultados de absorção de umidade nos compósitos condicionados em câmaras de climatização e de névoa salina.

\begin{tabular}{|c|c|c|c|c|c|c|c|c|c|}
\hline \multicolumn{10}{|c|}{ Ganho de massa médio (\%) } \\
\hline & $1^{\mathrm{a}} \operatorname{sem} . *$ & $2^{\mathrm{a}} \mathrm{sem}$. & $3^{\text {a }}$ sem. & $4^{\mathrm{a}} \mathrm{sem}$. & $5^{\mathrm{a}}$ sem. & $6^{\mathrm{a}} \mathrm{sem}$. & $7^{\mathrm{a}} \mathrm{sem}$. & $8^{\text {a }}$ sem. & $9^{\mathrm{a}}$ sem. \\
\hline \multicolumn{10}{|l|}{ Tração transversal } \\
\hline Câmara de climatização & 0,52 & 0,73 & 0,75 & 0,76 & 0,87 & 0,89 & 0,92 & 0,92 & 0,93 \\
\hline Névoa salina & 0,05 & 0,20 & 0,33 & 0,26 & 0,29 & 0,29 & 0,30 & 0,30 & 0,30 \\
\hline \multicolumn{10}{|l|}{ Tração longitudinal } \\
\hline Câmara de climatização & 0,55 & 0,70 & 0,71 & 0,62 & 0,62 & 0,61 & 0,62 & 0,62 & 0,62 \\
\hline Névoa salina & 0,22 & 0,38 & 0,38 & 0,35 & 0,36 & 0,37 & 0,37 & 0,37 & 0,37 \\
\hline
\end{tabular}

de difusão de Fick. A água permanece no compósito como água livre, e tende com o tempo a penetrar na resina pelo gradiente de concentração. Acima dessa porção linear, a absorção de umidade começa a conferir um formato côncavo na curva em relação ao eixo das abscissas, indicando um desvio positivo do estado de pseudo-equilíbrio de Fick. Com a contínua exposição, o processo de absorção de umidade se torna mais lento, e muitos autores atribuem a esse período, o início do processo de relaxação da cadeia polimérica, e o preenchimento higrotérmico dos vazios existentes ${ }^{[4,18-25]}$.

Nos compósitos condicionados em câmara de climatização, foi observado um ganho médio de massa entre $0,6 \%$ e $0,9 \%$. A absorção de umidade para os compósitos condicionados em câmara de névoa salina é de $0,25 \%$ e $0,35 \%$. Existe uma diferença no mecanismo de absorção de umidade nas direções paralelas e perpendiculares de amostras. Nos cdps usados para tração transversal a matriz polimérica fica mais exposta e assim, favorece a absorção de umidade. Já nos cdps usados para tração longitudinal, a presença de fibras nas bordas dificulta a absorção e difusão de umidade pelo compósito por isto apresenta valores menores de ganho de massa médio.

Quando se compara o ganho de massa médio dos compósitos condicionados em câmara de climatização à temperatura de $80{ }^{\circ} \mathrm{C}(0,6-0,9 \%)$ com aos submetidos em câmara de névoa salina à temperatura de $35^{\circ} \mathrm{C}(0,25-0,35 \%)$, observa-se que os primeiros apresentam um ganho de massa maior. Essa maior absorção de umidade por parte dos compósitos submetidos à câmara de climatização deve-se ao fato da temperatura utilizada na câmara de climatização favorecer uma maior absorção de umidade pelos compósitos.

\section{Comportamento em tração longitudinal}

Os ensaios de resistência à tração longitudinal dos compósitos fabricados com fita unidirecional de fibra de carbono IM7 impregnada com resina epóxi 8552 foram realizados para determinar a propriedade do compósito pela medida de resistência à tração de fibras unidirecionais posicionadas no sentido do carregamento. Ao contrário do que ocorre no ensaio de tração transversal, onde o esforço é dominado pela resina, no ensaio de tração longitudinal o esforço é dominado pela fibra.

Os resultados apresentados na Tabela 4 e ilustrados na

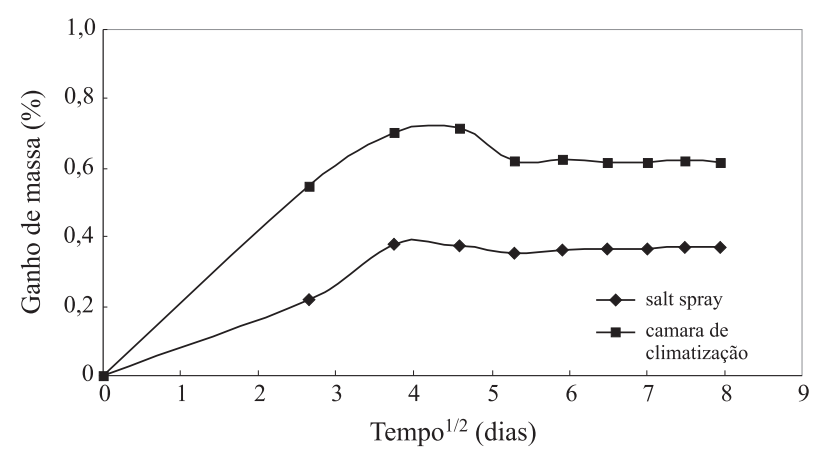

Figura 4. Ganho de massa médio do compósito para tração longitudinal condicionado em câmaras de climatização e de névoa salina.

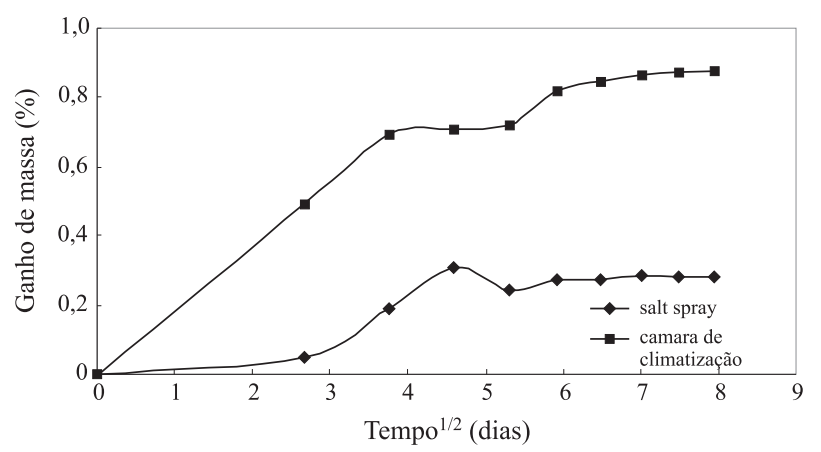

Figura 5. Ganho de massa médio do compósito para tração transversal condicionado em câmaras de climatização e de névoa salina.

Figura 6, para os cdp's condicionados e não condicionados, são os valores das médias de resistência à tração das amostras ensaiadas.

As resistências à tração longitudinal, determinadas para os cdp's (Tabela 4) ensaiados na condição ambiente (2627,0 \pm $116,6) \mathrm{MPa}$, mostram que os compósitos não reduzem sua resistência quando condicionados em câmara de névoa salina $(2615,2 \pm 84,7) \mathrm{MPa}$. Estes resultados foram comprovados através de método estatístico de comparação de duas médias, utilizando o segundo caso de dados não-emparelhados, com uma probabilidade de $95 \%{ }^{[26]}$. Mas as amostras submetidas à câmara de climatização $(2478,2 \pm 39,5) \mathrm{MPa}$ apresentam uma redução de 5,7\% na resistência à tração longitudinal, quando comparadas com as amostras não condicionadas.

Os valores médios de resistência à tração longitudinal dos cdp's (Tabela 4) ensaiados em temperatura elevada $\left(82^{\circ} \mathrm{C}\right)$ 
Tabela 4. Resultados dos ensaios de resistência à tração longitudinal.

\begin{tabular}{lccc}
\hline $\begin{array}{c}\text { Ensaios à temperatura } \\
\text { ambiente }\end{array}$ & $\begin{array}{c}\text { Máximo } \\
(\mathbf{M P a})\end{array}$ & $\begin{array}{c}\text { Mínimo } \\
(\mathbf{M P a})\end{array}$ & Médio $(\mathbf{M P a})$ \\
\hline Sem condicionamento & 2808,8 & 2462,4 & $\mathbf{2 6 2 7 , 0} \pm \mathbf{1 1 6 , 6}$ \\
Câmara de climatização & 2551,1 & 2446,8 & $\mathbf{2 4 7 8 , 2} \pm \mathbf{3 9 , 5}$ \\
Câmara de névoa salina & 2739,1 & 2533,4 & $\mathbf{2 6 1 5 , 2} \pm \mathbf{8 4 , 7}$ \\
\hline \multicolumn{1}{c}{ Ensaios em } & $\begin{array}{c}\text { Máximo } \\
\text { temperatura elevada }\end{array}$ & $\begin{array}{c}\text { Mínimo } \\
(\mathbf{M P a})\end{array}$ & Médio $(\mathbf{M P a})$ \\
\hline Sem condicionamento & 2591,5 & 2476,6 & $\mathbf{2 5 3 1 , 5} \pm \mathbf{4 0 , 4}$ \\
Câmara de climatização & 1950,1 & 1713,7 & $\mathbf{1 8 3 2 , 7} \pm \mathbf{9 2 , 3}$ \\
Câmara de névoa salina & 2644,4 & 2315,5 & $\mathbf{2 4 9 8 , 3} \pm \mathbf{1 3 4 , 1}$ \\
\hline
\end{tabular}

demonstram que os compósitos condicionados em câmara de névoa salina $(2498,3 \pm 134,1) \mathrm{MPa}$ reduzem em $1,3 \%$ sua resistência em relação aos sem condicionamento $(2531,5 \pm$ 40,4)MPa. Estes resultados foram comprovados através de método estatístico de comparação de duas médias utilizando o segundo caso de dados não-emparelhados, com uma probabilidade de $95 \%{ }^{[26]}$. Porém, os compósitos condicionados em câmara de climatização $(1832,7 \pm 92,3) \mathrm{MPa}$ apresentam uma redução de $27,6 \%$ na sua resistência, indicando que os compósitos quando submetidos à saturação em ambiente com $90 \%$ de UR e $80^{\circ} \mathrm{C}$, sofrem uma maior influência da temperatura e umidade, caracterizando um ambiente mais agressivo ao compósito.

Comparando-se os resultados dos compósitos ensaiados em condição ambiente e em temperatura elevada (Tabela 4 e Figura 6), constata-se que as amostras não condicionadas e ensaiadas à temperatura elevada $(2531,5 \pm 40,4) \mathrm{MPa}$ apresentam uma pequena redução de $3,6 \%$ na sua resistência em relação às não condicionadas e ensaiadas à temperatura ambiente $(2627,0 \pm$ 116,6)MPa. Quando comparados os resultados das amostras condicionadas em câmara de névoa salina e ensaiadas à temperatura ambiente $(2615,2 \pm 84,7) \mathrm{MPa}$ com as ensaiadas a temperatura elevada $(2498,3 \pm 134,1) \mathrm{MPa}$, observa-se que os compósitos ensaiados à temperatura elevada têm uma perda

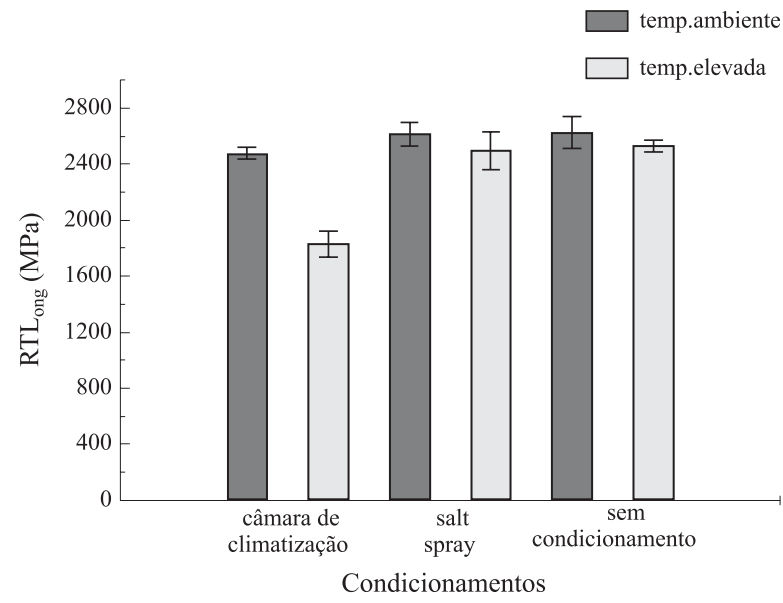

Figura 6. Resistência à tração longitudinal às temperaturas ambiente e elevada ( $\mathrm{RTL}_{\text {ong }}$ ). de 4,5\% na sua resistência em relação aos ensaiados à temperatura ambiente. Analisando-se os resultados das amostras condicionadas em câmara de climatização, verifica-se que os compósitos ensaiados à temperatura ambiente $(2478,2 \pm$ $39,5) \mathrm{MPa}$ são em média $26 \%$ mais resistentes do que os compósitos ensaiados à temperatura elevada $(1832,7 \pm$ 92,3)MPa. Reafirmando, mais uma vez, que o efeito combinado da temperatura elevada de ensaio com o condicionamento em câmara de climatização controlada, reduz a resistência à tração longitudinal, devido à degradação da matriz polimérica e/ou da interface fibra/matriz.

\section{Comportamento em tração transversal}

As medidas das resistências à tração das famílias de compósitos unidirecionais sem condicionamento e condicionados em câmara de climatização e de névoa salina, estão apresentadas na Tabela 5 e ilustrados na Figura 7. O desvio padrão, os valores máximo e mínimo medidos também são incluídos para caracterizar a dispersão de dados. Este parâmetro estatístico nas medidas da resistência à fratura é fundamental para indicar se o processo de fabricação do compósito é consistente. Além disso, tem uma função crítica quando utilizado na definição de valores de resistência de projeto para a fabricação de peças estruturais ${ }^{[13]}$.

A resistência à tração transversal dos cdp's (Tabela 3) ensaiados na condição ambiente $\left(22^{\circ} \mathrm{C}\right)$, mostra que os compósitos submetidos à câmara de névoa salina $(53,1 \pm$ $3,7) \mathrm{MPa}$ apresentam uma diminuição de $13,5 \%$ na sua resistência. As amostras submetidas à câmara de climatização $(36,0 \pm 2,2) \mathrm{MPa}$ apresentam uma redução de $41,4 \%$ em sua resistência à tração transversal, quando comparadas com as amostras não condicionadas $(61,4 \pm 1,9) \mathrm{MPa}^{[6,27]}$.

Comparando-se os dados encontrados com os resultados obtidos por Bao e Yee ${ }^{[28]}$, pode-se confirmar o efeito danoso que a temperatura exerce sobre os compósitos ensaiados em câmara de climatização, uma vez que se observa uma degradação maior na região da matriz polimérica sobre o efeito da temperatura elevada. Como mencionado anteriormente, no ensaio de tração transversal o esforço é dominado pela resina, onde as fibras estão orientadas perpendicularmente ao eixo de solicitação do esforço. Além disto, há alojamento de

Tabela 5. Resultados dos ensaios de resistência à tração transversal.

\begin{tabular}{lccc}
\hline $\begin{array}{c}\text { Ensaios à } \\
\text { temperatura ambiente }\end{array}$ & $\begin{array}{c}\text { Máximo } \\
(\mathbf{M P a})\end{array}$ & $\begin{array}{c}\text { Mínimo } \\
(\mathbf{M P a})\end{array}$ & Médio (MPa) \\
\hline Sem condicionamento & 64,5 & 59,3 & $\mathbf{6 1 , 4} \pm \mathbf{1 , 9}$ \\
Câmara de névoa salina & 59,6 & 48,3 & $\mathbf{5 3 , 1} \pm \mathbf{3 , 7}$ \\
Câmara de climatização & 38,8 & 33,6 & $\mathbf{3 6 , 0} \pm \mathbf{2 , 2}$ \\
\hline Ensaios em & Máximo & Mínimo & Médio $(\mathbf{M P a})$ \\
temperatura elevada & (MPa) & (MPa) & \\
\hline Sem condicionamento & 52,4 & 43,6 & $\mathbf{4 7 , 6} \pm \mathbf{3 , 6}$ \\
Câmara de névoa salina & 45,1 & 39,0 & $\mathbf{4 2 , 4} \pm \mathbf{2 , 0}$ \\
Câmara de climatização & 25,9 & 21,1 & $\mathbf{2 3 , 4} \pm \mathbf{1 , 8}$ \\
\hline
\end{tabular}


umidade na interface fibra-matriz o que causa sua degradação e a diminuição da resistência longitudinal e transversal, bem como a matriz pode absorver umidade provocando um inchamento da mesma que por sua vez causa tensões internas no compósito reduzindo a sua resistência a tração transversal.

Comportamento similar é observado nas resistências à tração transversal medidas nos cdp's ensaiados à temperatura elevada $\left(82^{\circ} \mathrm{C}\right)$ (Tabela 5), demonstrando que os compósitos submetidos à câmara de névoa salina $(42,4 \pm 2,0) \mathrm{MPa}$ apresentam uma redução de $10,9 \%$ na sua resistência, quando comparados com os sem condicionamento $(47,6 \pm 3,6) \mathrm{MPa}$. Uma drástica diminuição da resistência à tração transversal de $50,8 \%$, é observada quando as amostras de compósitos poliméricos foram submetidas à câmara de climatização e ensaiadas à temperatura elevada $(23,4 \pm 1,8) \mathrm{MPa}$. Estes fatos comprovam mais uma vez que a matriz polimérica é severamente afetada pelos ensaios em temperatura elevada.

Comparando-se os resultados dos compósitos ensaiados sem condicionamento, ensaiados em condição ambiente e em temperatura elevada (Tabela 5 e Figura 7), constata-se que as amostras não condicionadas e ensaiadas em temperatura elevada $(47,6 \pm 3,6) \mathrm{MPa}$ apresentam uma diminuição de 22,5\% em relação às não condicionadas e ensaiadas à temperatura ambiente $(61,4 \pm 1,9) \mathrm{MPa}$, ficando evidente que a temperatura tem influência nas propriedades mecânicas dos compósitos. Quando comparados os resultados das amostras condicionadas em câmara de névoa salina e ensaiadas à temperatura ambiente com as ensaiadas à temperatura elevada, observa-se que os compósitos ensaiados à temperatura elevada $(42,4 \pm 2,0) \mathrm{MPa}$ apresentam uma redução de 20,2\% na resistência em relação aos ensaiados à temperatura ambiente $(53,1 \pm 3,7) \mathrm{MPa}$. Analisando-se os resultados das amostras condicionadas em câmara de climatização, verifica-se que os compósitos ensaiados à temperatura ambiente $(36,0 \pm$ $2,2) \mathrm{MPa}$ são em média $35 \%$ mais resistentes do que os compósitos ensaiados à temperatura elevada $(23,4 \pm 1,8) \mathrm{MPa}$. Todos estes dados confirmam que o efeito combinado da elevada temperatura de ensaio com o condicionamento em câmara

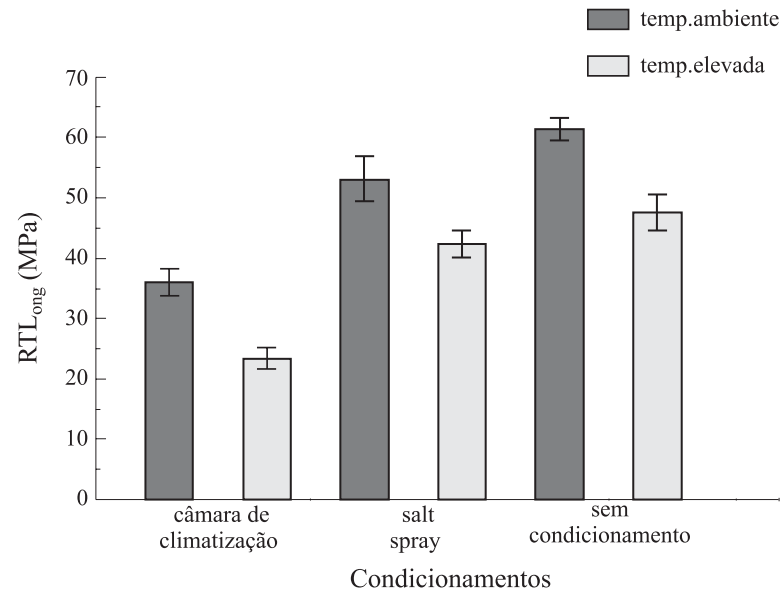

Figura 7. Resistência à tração transversal às temperaturas ambiente e elevada $\left(\mathrm{RT}_{\text {transs }}\right)$. de umidade controlada, reduz as resistências à tração transversal, devido à degradação da matriz polimérica.

\section{Microscopia eletrônica de varredura}

Sabendo-se que, uma boa adesão entre a fibra e a resina é pré-requisito para uma boa transferência de tensões nos compósitos, a análise do modo de falha interfacial suportado pelo conhecimento da qualidade da adesão fibra/resina deve ser uma condição essencial para explicar a resistência à tração dos compósitos. No caso da absorção de água pelos compósitos, os resultados obtidos devem ser dependentes das propriedades da interface fibra/resina tanto quanto da própria resina.

Sendo assim, na tentativa de explicar a influência do condicionamento ambiental no mecanismo de adesão interfacial fibra/resina dos compósitos utilizou-se a técnica de microscopia eletrônica de varredura (MEV). Utilizando-se microscópios eletrônicos de varredura LEO modelo $435 \mathrm{Vpi}$ e Zeiss modelo DSM 950.

A análise da região interfacial fibra/resina é realizada no plano de fratura dos compósitos sem condicionamento, condicionados em câmaras de climatização e de névoa salina, bem como ensaiados às temperaturas ambiente e elevada.

Observa-se que os cdp's ensaiados em tração longitudinal apresentam uma ruptura de difícil análise do ponto de vista fractográfico, uma vez que se fragmentaram impedindo que fosse realizada uma detalhada investigação morfológica da região fraturada, como apresentada na literatura por Franco ${ }^{[6]}$. Já os cdp's ensaiados em tração transversal apresentam uma nítida ruptura transversal na direção de carregamento, permitindo que fosse realizada uma detalhada investigação morfológica da região fraturada.

Comparando-se as superfícies de fratura dos cdp's sem condicionamento, ensaiados às temperaturas ambiente e elevada, observa-se que os compósitos submetidos à temperatura ambiente mostram a presença de resina aderida à superfície das fibras, apresentando uma região de ancoragem da matriz na fibra bem definida e rica em resina, textura essa que é denominada de cusps $^{[6,29,30]}$, demonstrando que não ocorre deslocamento das fibras e revelando que o rompimento das amostras durante o ensaio ocorre na resina. Os compósitos ensaiados em temperatura elevada apresentam uma fina camada de resina cobrindo a superfície das fibras, mas as regiões ricas em resina mostram-se, em sua maioria, rompidas, deixando resina solta sobre a fibra do compósito. Tal observação é atribuída à degradação da matriz, causada pelo efeito deletério da temperatura de ensaio.

As figuras a seguir mostram a superfície de fratura dos compósitos após nove semanas de condicionamento em câmara de climatização. A Figura 8 mostra o compósito ensaiado à temperatura ambiente e condicionado em câmara de climatização, onde as superfícies lisas das fibras indicam a ocorrência de deslocamento interfacial nesta região durante o ensaio. Na Figura 9 é apresentado o compósito ensaiado à temperatura elevada e condicionado em câmara de climatização, que mostra a imagem das superfícies das fibras lisas, 


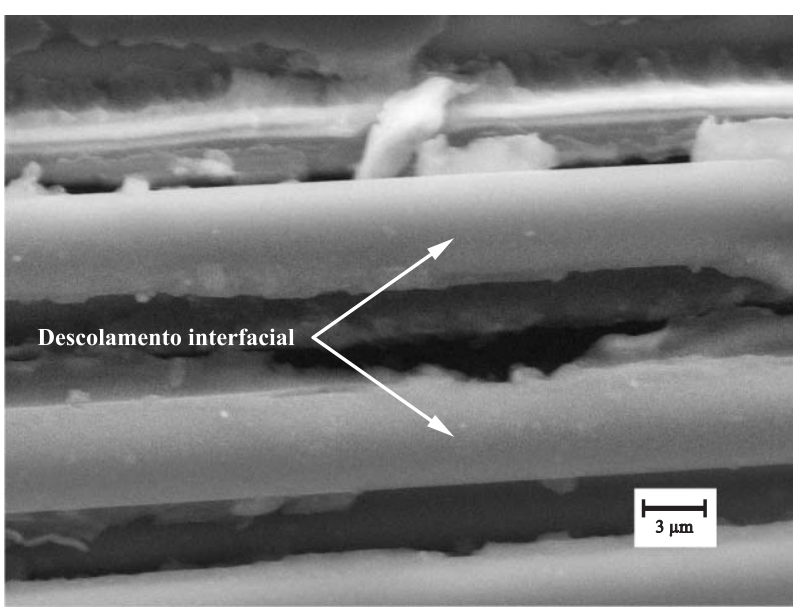

Figura 8. Compósito condicionado em câmara de climatização e ensaiado em tração transversal à temperatura ambiente.

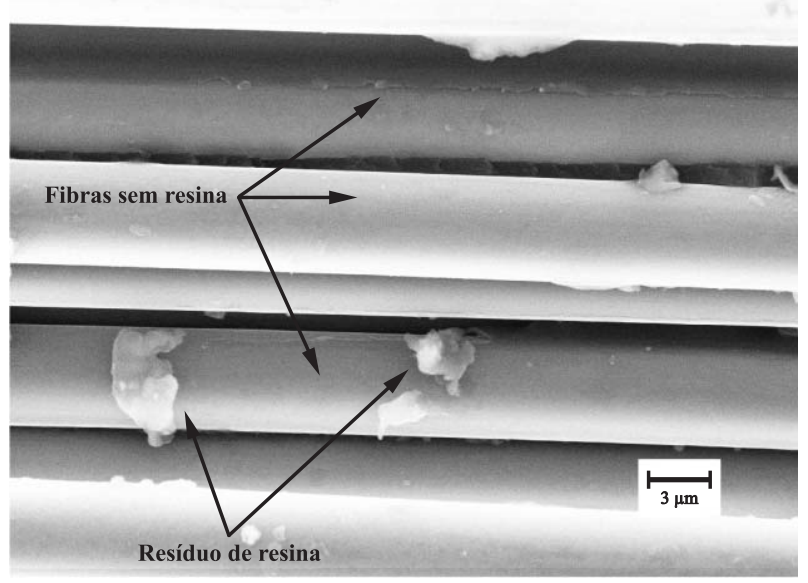

Figura 9. Compósito condicionado em câmara de climatização e ensaiado em tração transversal em temperatura elevada

praticamente sem resina, com resíduos da matriz polimérica soltos no plano de fratura.

Quando é feita uma comparação entre as superfícies de fratura dos compósitos após nove semanas de condicionamento em câmara de névoa salina, observa-se que as amostras ensaiadas à temperatura ambiente possuem na região analisada aspectos tipo cusps na resina entre algumas fibras, regiões estas com o rompimento da interface fibra/resina e fibras cobertas com uma fina camada de resina. Já na micrografia dos compósitos ensaiados em temperatura elevada é observada a ausência de cusps na resina em torno das fibras e o rompimento da interface fibra/resina, além de apresentar descontinuidade na fina camada que recobre as fibras, indicando a perda de resina na superfície do reforço.

\section{Conclusão}

$\mathrm{O}$ estudo dos efeitos higrotérmicos sobre a resistência à tração de compósitos de fita de carbono unidirecional impregnadas com resina epóxi 8552 mostra que a matriz polimérica é severamente afetada nos ensaios em temperatura elevada. Os ensaios de resistência à tração longitudinal, realizados à temperatura ambiente, mostraram que as amostras não perderam sua resistência quando condicionadas. Porém, os compósitos apresentaram uma redução na sua resistência quando submetidos à câmara de climatização e ensaiados à temperatura elevada. Já nos ensaios de tração transversal foi observado que as amostras submetidas à câmara de névoa salina e ensaiadas à temperatura ambiente apresentaram uma pequena redução na sua resistência, porém as amostras submetidas à câmara de climatização mostraram uma redução acentuada na resistência As amostras ensaiadas à temperatura elevada e submetidas à câmara de névoa salina apresentaram uma pequena diminuição na sua resistência (11\%). Já uma acentuada diminuição da resistência à tração transversal (51\%) foi observada quando as amostras de compósitos poliméricos foram submetidas à câmara de climatização e ensaiadas à temperatura elevada. Assim sendo, o efeito combinado da temperatura elevada de ensaio com o condicionamento em câmara de umidade controlada, reduz as resistências à tração transversal e longitudinal, devido à degradação da matriz polimérica.

\section{Agradecimentos}

Os autores agradecem ao SENAI - SP, à EMBRAER por fornecer todo o material utilizado no presente trabalho, à FAPESP (02/01808-7) e ao CNPq (303528/2003-6).

\section{Referências Bibliográficas}

1. Marshall, A C. - "Composite structures in home built sport aircraft", in: Proceedings of International Sampe Symposium,32 ${ }^{\text {nd }}$, p.1510 (1987).

2. Hergenrother, P. M. -SAMPE Journal, 36, jan./feb. p. 30 (2000).

3. Morgan, R. J. - SAMPE Journal, 37, p. 102 (2001).

4. Costa, M. L. - "Efeito do conteúdo de vazios no comportamento mecânico de compósitos avançados carbono/epóxi e carbono/bismaleimida". Tese de Doutorado, Instituto Tecnológico de Aeronáutica, São José dos Campos (2002).

5. Barbosa, S. C. - "Comportamento mecânico e análise de danos de materiais compósitos obtidos pelo processo de pultrusão". Tese de Mestrado, Instituto de Tecnológico da Aeronáutica, São José dos Campos (1995).

6. Franco, L. A. L. - "Análise fractográfica de compósitos poliméricos estruturais". Tese de Mestrado, Instituto Tecnológico de Aeronáutica, São José dos Campos (2003).

7. Mayer, S. - "Influência do condicionamento ambiental na resistência à tração de laminados de compósitos poliméricos reparados". Tese de Mestrado, Instituto Tecnológico de Aeronáutica, São José dos Campos (2003). 
8. Kelly, A; Mileiko, S. T. (Ed.). - "Fabrication of composites". North-Holland: Elsevier North-Holland, 574p. (Handbook of Composites, v. 4) (1983).

9. Strong, A. B. - "Fundamentals of composite manufacturing: materials, methods and applications". (S.1.): Society of Manufacturing Engineers (1989).

10. Demonte, J. D. - "Technology of carbon and graphite fiber composites". Malabar, FL: Robert E. Krieger, cap. 9 (1987).

11. Soles, C.L.; Chang, F.T.; Bolan, B.A.; Hristov, H.A.; Gidley, D.W.; Yee, A.F. - Journal of Polymer Science: Part B: Polymer Physics, 36, p.3035 (1998).

12. Delaware Composite Design Encyclopedia -"Processing and Fabrication Technology", Technomic Publishing Co. Inc, USA (1990).

13. Thomason, J. L. - Composites, 26, p. 475 (1995).

14. Tajima, Y. A. SAMPE Quaterly, jul, p. 1 (1980).

15. Kondo, K; Taki, T.- "Moisture diffusivity of unidirectional composites", in: Springer, G. S. (Ed) Environmental Effects on Composite Materials., Technomic,.v. 2, cap.24, Lancaster (1984)

16. Ashbee, K. H. G. - "Fundamental principles of fiber reinforced composites". 2nd. ed. Lancaster, PA: Technomic, cap.10 (1993).

17. Wolff, E.G. - SAMPE Journal, 9, p.11 (1993).

18. Cândido, G. M. - "Influência do condicionamento ambiental na resistência a delaminação de borda livre em compósitos avançados". Tese de Doutorado, Instituto Tecnológico de Aeronáutica, São José dos Campos (2001).

19. Shen, C.H.; Springer, G. S. - "Moisture absorption and desorption of composite materials", in Springer, G. S. (Ed) Environmental Effects on Composite Materials., Technomic, 1981. v. 1, cap.3, Westport (1981).

20. Bradley, W.L.; Grant, T. S. Journal of Materials Science, 30, p. 5537 (1995).

21. De Neve, B, Shanahan, M.E.R. Polymer, 34, p. 5099 (1993).

22. Nuñez, L. et al. Journal of Applied Polymer Science, 74, p. 353 (1999).

23. Collings, T. A.; Harvey, R. J.; Dalziel, A.W. Composites, 24, 8, p. 625 (1993).
24. Catálogo Hexcel, 2002.

25. American Society For Testing And Materials. ASTM C562-85: "Standard test method for moisture in a graphite sample" (1985).

26. American Society For Testing And Materials. D 5229/D 5229M-04: "Moisture absorption properties and equilibrium conditioning of polymer matrix composite materials" (2004).

27. American Society For Testing And Materials. D 117-03: "Salt spray (fog) testing" (2003).

28. American Society For Testing And Materials. ASTM D 3039/D 3039M-00: "Tensile properties of fiber-resin composites" (2000).

29. Ishai, O. - Polymer Engineering and Science, 15, p. 491 (1975).

30. Harper, B.D.; Staab, G. H.; Chen, R.S. - Journal Composite Materials, 21, p.280 (1987).

31. Chateauminois, A; Vicent, L. - Polymer, 35, p.22 (1994).

32. Paplham, W.P.; Brown, R.A.; Salin, I.M.; Seferis, J.C. Journal of Applied Polymer Science, 57, p.133 (1995).

33. Thomason, J. L. - Composites, 26, p. 475 (1995 ).

34. Cândido, G.M.; Rezende, M.C.; Almeida, S.F.M. Materials Research, 3, p. 11 (2000).

35. Choi, H.S.; Ahn, K.J.; Nam, J.D.; Chun, H.J. - Composites Part A: applied science and manufacturing, 32, p.709 (2001).

36. Costa Neto, P. L. O. - "Estatística”., Ed. Edgard Blücher, p. 108, cap. 5 São Paulo (1977).

37. Selzer, R.; Friedrich, K. - Composites Part A: Applied Science And Manufacturing,. 28A, p. 595 (1997).

38. Bao, L.; Yee, A. F. - Composites Science and Technology, 62, p. 2099 (2002).

39. Purslow, D. - Composites. 17, p. 289 (1986).

40. Greenhalgh, E.; Hiley, M.; Singh, S. - "Introduction to fractographic analysis if composites". Course Notes, Structural Materials Centre, DERA, Farnborough Hants, UK. Oct. (1997).

Enviado: 23/06/05

Reenviado: 02/03/06

Aprovado: 17/03/06 